\title{
Optic Disc Segmentation based on Template Matching and Active Contour Method
}

\author{
S. A. TUNCER
}

\begin{abstract}
This paper proposes a hybrid method that is capable of automatically implementing the Optic Disc (OD) segmentation. In the hybrid method, two steps were performed. First, the location of the OD was determined by template matching. Second, the OD location coordinates obtained in the first stage were given as inputs to the Active Contour Method applied to complete the OD segmentation. Furthermore, as part of this study, Android based a program was developed to allow physicians the ability to independently access the proposed hybrid method results from anywhere and to add comments. Thus, the physician would be able to instantly track the patient. Performance evaluation of the proposed hybrid method was done separately for both localization and segmentation. The success of localization was confirmed on the basis of whether the determined coordinates corresponded to the OD. The segmentation process was assessed according to the parameters, as derived from a confusion matrix. The average Dice coefficient obtained for all images was $\mathbf{0 . 9 4 3}$, while the average values of accuracy, specificity and sensitivity parameters for all images were calculated as $0.90,0.961$ and 0.931 , respectively. The final results obtained from the proposed hybrid method were checked by a physician, who observed that the OD was successfully segmented.
\end{abstract}

Index Terms - Active Contour, Optic Disc, Template Matching

\section{INTRODUCTION}

$\mathrm{T}$ HANKS to the advancements made in computerized techniques, preliminary diagnoses and regular diagnoses of diseases can be made using retinal images. Detection of impairments on structures, such as veins and Optic Disc (OD), in retinal images is crucial for disease diagnosis and followup. For example, diabetic retinopathy, age-related macular degeneration (ARMD), and glaucoma are included among the diseases able to be detected from degenerations on retinal images. The first step in the detection of these diseases is to examine the OD. In identifying abnormalities on the OD, false diagnoses can be made due to physical-related issues involving the physician, such as fatigue. Automatic systems are therefore needed to reduce the error rate and the workload of physicians and to speed up the identification of parameters for diagnosis of the disease. The development and use of

SEDA ARSLAN TUNCER, is with Department of Software Engineering University of Firat, Elazig, Turkey, (e-mail: satuncer@ firat.edu.tr).

(iD) https://orcid.org/0000-0001-6472-8306

Manuscript received October 15, 2018; accepted January 30, 2019

DOI: $\underline{10.17694 / \text { bajece. } 470796}$ automated systems will serve to significantly reduce the workload of the healthcare personnel who are responsible for visually examining and analyzing very large volumes of data [1].

The OD is the part of the optic nerve that is attached to the retina. It is usually located in the middle of the eye and has a yellowish, bright and round appearance, and nerve heads. The OD is not light sensing and is positioned in an area where the blood vessels pass through. In retinal images, the average OD diameter is $1.75 \mathrm{~mm}$ in the vertical and $1.5 \mathrm{~mm}$ in the horizontal [2]. The OD structure on the retina is shown in Figure.1.

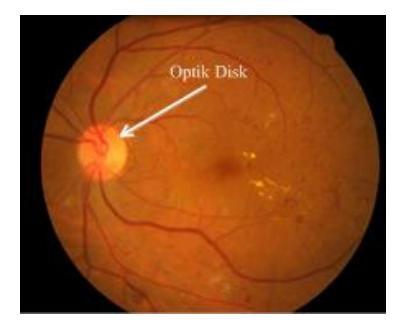

Fig.1. OD in Retinal image

OD segmentation from retinal images is the first step in diagnosing and following retinal diseases. In this paper, a hybrid method for performing OD segmentation is proposed to detect and follow retinal diseases. The proposed hybrid method involves 2 steps. In the first step, the OD location is determined using the template matching method. In the second step, OD segmentation is performed by submitting the location information of each OD as input for the Active Contour Method (ACM). In addition, a web-based system was developed to allow physicians the capability of following-up the patient in the mobile environment. An Android-based interface was created to access this developed system and obtain segmentation results.

This paper shall serve to contribute the following to the literature:

-The ACM, which is a semi-automatic method, was converted to a fully automatic method using template matching. In this way, the OD is more easily segmented.

-An android-based structure was developed to evaluate the disease. With this structure, the physician can independently access the proposed hybrid method results from anywhere and add comments.

This paper, whose focus is on optic segmentation, is organized in 6 sections, with the section above being the first. 
The second section presents a literature review on OD segmentation. In the third section, the properties of the retinal image to be used in practice are given, and the process of conversion to a fully automatic method of the ACM and the proposed hybrid system for OD segmentation are presented. Section 5 provides an explanation of the proposed Android system framework. In Section 6, obtained results are discussed. And finally, the advantages of the proposed hybrid method are mentioned and talked about future work.

\section{RELATED WORKS}

In the literature, there are a number of pixel-based or modelbased studies on the segmentation of OD on retinal images [3, $4,5]$. When examining the research conducted on determining the OD location, it was observed that many image processing techniques, such as morphological operations, edge extraction filters, segmentation, Hough transform, and thresholding, are used. Studies related to this subject have largely focused on determining the location or edge segmentation of the OD in the retinal image. In determining the location, the aim is to identify the OD center and the OD boundary with minimum error, while in the segmentation process; the challenge is distinguishing the OD from other structures in the eye.

A study by $\mathrm{Li}$ et al. converted retinal images to a pixel-based gray level, where regions with bright pixels were identified as candidate clusters, and these clusters were then determined using a model-based method best suited for the OD [4]. In another study using color morphology, vessels were removed from the image using the automatically activated ACM, and the image was made ready for analysis [5]. A study focusing on diagnosing glaucoma disease, detection of the OD was performed by automatic thresholding after the noise was eliminated, and the brightness was normalized for automatic segmentation [6]. In [7], the retinal image was first converted to gray scale using the Basic Component Analysis method. The Stochastic Watershed algorithm was then applied to this transformed image to perform OD localization. In 110 eye images, the OD location was determined with an accuracy of $86.89 \%$. In [8], authors conducted a study where they used the Haar Wavelet Transformation method to determine the location of the OD over the green color channel, and the edge extraction was performed by applying the Hausdorff-based matching method. In this study, the success rate was obtained $93 \%$ over 40 images. In [9], authors obtained edges of ODs by applying the Sobel Edge Removal Filter to the retinal image. In the same study, by applying the Circular Hough Transformation on this extracted image, they were able to detect the OD location, and a $97 \%$ success rate was achieved over 40 images. The literature shows that hybrid methods have been proposed for automatic segmentation of the OD $[10,11,12]$. For example, In [10] was developed a fully automated method using the region growing method and the L1 minimization algorithm, which are semi-automatic methods for obtaining OD segmentation. With the proposed hybrid method in Tuncer's study, there was $92 \%$ and $87 \%$ similarity according to the similarity indices of Dice and Jaccard, respectively. In [11], authors segmented the OD using the hybrid vessel phase portrait analysis, where the results showed that true negatives and true positives were obtained in 94.67 and $98.13 \%$ of cases, respectively. In [12], OD was segmented with $98.67 \%$ success using a hybrid approach involving the ACM and ellipse fit method. In [13], OD was determined by histogram matching technique to identify the presence of pathological regions. Success rates for used data sets were $100 \%, 98.9 \%$, and $91.36 \%$ for the datasets, respectively. In [14], authors proposed an alternative technique for automatic segmentation of OD. The technique is based on a circular transformation which was implemented to detect circular boundary and colour variation. Technique was applied to the three data sets. Accuracy for data sets was $98.77 \%, 97.5 \%$ and $99.75 \%$, respectively. In [15], the OD location was first determined by the brightness-based method. Then supervised descent method was used to segment the optical disc. The proposed method has compared with the circular Hough transform, the directional matched filtering and level-sets method, the principal component analysis and mathematical morphology based method, the sliding band filter method, the snake-based contour refinement method and the super pixel-based classification method. The results showed that the proposed approach outperformed the existing methods. In [16], authors have presented a novel method to automatically segment $\mathrm{OD}$ in fundus images based on variational model with multiple energies. Firstly, to localize the OD center has used a sparse coding based technique. Next, OD segmentation has been regarded as an energy minimization problem, and a variational model integrating three energy terms has been proposed to evolve the curve to the OD boundary. Experimental results demonstrate that the proposed method outperforms the state-of-the art techniques. In [17], a fully automatic method for the OD segmentation in retinal images has proposed. This method is a multiresolution sliding band filter (SBF). An average overlapping area of $83 \%, 89 \%$ and $85 \%$ has achieved for the images in three datasets, respectively. In [18] was developed and trained a convolutional neural network to automatically and simultaneously segment optic disc, fovea and blood vessels. The results shown that, OD were segmented with $92.68 \%$ accuracy. In [19], an entropy-based method was proposed to determine the OD location. Five data sets were used and the accuracy was determined between $75 \%$ and $100 \%$.

\section{MATERIAL}

The retinal images used in this paper were obtained from Firat University Department of Ophthalmology retina database. A total of 40 retinal images were used for segmentation, with the images being in $45^{\circ} \mathrm{FOV}$ and jpeg format. Both healthy and diseased images were randomly selected from the data on the right and left eyes. The resolution of each image was $565 \times$ 584 pixels.

\section{PROPOSED HYBRID METHOD}

The hybrid method for fully automated OD segmentation procedure that was proposed in this paper is shown in Figure 2. To carry out the hybrid method, first, pre-processing was 
done for each image, such as filtering and gamma conversion. Next, the template matching method was applied to determine OD location. The obtained location information was then used as input to the ACM for segmentation. Lastly, a performance evaluation was conducted to determine the accuracy of the OD segmentation.

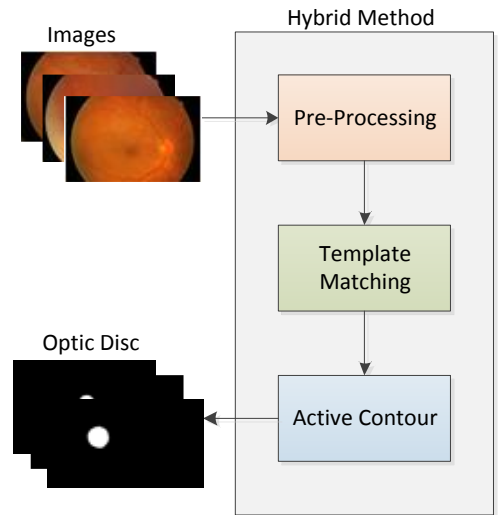

Fig.2. Proposed Hybrid Method for OD segmentation

\section{A. Template Matching}

Template Matching is a method for searching and finding the location of a template image in a larger image. The template image is routed over the large image and similarity is measured by mapping on each pixel. The first step for the detection of pathological areas in the OD is segmentation. For this, template matching algorithm is applied after preprocessing step. In the proposed hybrid system, five retinal images were used as templates to identify the OD. For the template, three histograms were created, each corresponding to a color component. A histogram is a graph that shows the number of pixels at different density values in an image. An median filter was applied to the retinal images to reduce the noisy effect in the images. Then, a window with an OD size of $80 \times 80$ pixels was used to remove the OD from each retinal image. In the next step, the histogram of each color component was obtained by separating each OD color component (red, blue and green). Finally, for all retinal image samples, the average histogram of each color component was used as a template and the OD location was determined. The process for determining the OD location can be explained in detail as follows.

Each moving window channel (red, blue and green) is separated and the histogram of each channel is obtained. Then, the correlation between the histogram of each channel in the moving window and the histogram of the corresponding channel in the template is calculated. The function used for the correlation between two histograms is expressed as in equation 1.

$$
c=\frac{1}{\left(1+\sum_{i}\left(a_{i}-b_{i}\right)^{2}\right)}
$$

where $a$ and $b$ are the two histograms for which we want to calculate the correlation. The correlation used to determine template and OD similarity and relationship of force takes value at 0-1 interval. If two histograms ( $a$ and $b)$ are completely similar, then $c$ is equal to 1 otherwise 0 . Figure 3 shows a histogram example of one of the data used as template.

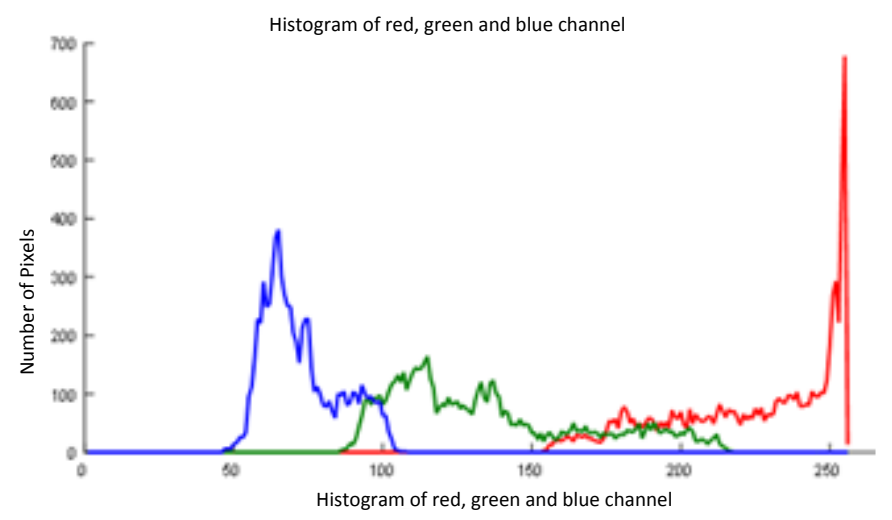

Fig.3 r, g, b histogram values for an image used as a template

For each moving window, we obtain three values as the results of correlation between the histograms. The result of histograms matching is computed as the weighted sum of the three obtained values:

$$
C(i, j)=t_{r} x c_{r}+t_{g} x c_{g}+t_{b} x c_{b}
$$

where $(i, j)$ is the center of moving window. $c_{r}, c_{g}$, and $c_{b}$ are the results of correlation for three channels (red, green, and blue) and $t_{r}, t_{g}$, and $t_{b}$ are weights used for each channel. In Equation (2), we can use different weights for $c_{r}, c_{g}$, and $c_{b}$. The green channel has the highest weight because the contrast of the green channel is higher than red and blue channels $[27,13]$. In some retinal images, blue channel is noisy; therefore, to decrease the effect of blue channel on our localizing method, we determine the lowest weight for blue channel. The best weights that result high accuracy rate for OD localizing method are $t_{r}=0.5, t_{g}=2$, and $t_{b}=1$. To localize the center of OD, we apply thresholding on the correlation function $C(i, j)$. For finding the best threshold, we did a global scanning of different values and the best equation to determine the threshold $\left(T_{h}\right)$ was obtained as follows.

$$
\mathrm{T}_{\mathrm{h}}=0.5 * \max (\mathrm{C})
$$

where $\max (C)$ is the element of $C$ with the maximum value. Therefore, the threshold value for each image is half of the maximum value of the correlation function. The center of gravity of the binary image obtained from thresholding is considered as the center of OD. Three retina images used to remove the OD histograms in the data set are shown in figure.4.

The specified OD coordinates are marked with the $x$ symbol. Coordinate information of OD is given as an input to the Active Contour Method which is a semi-automatic method. Thus, thanks to the proposed hybrid method, region of interest, namely the OD, is obtained by a fully automatic method. 


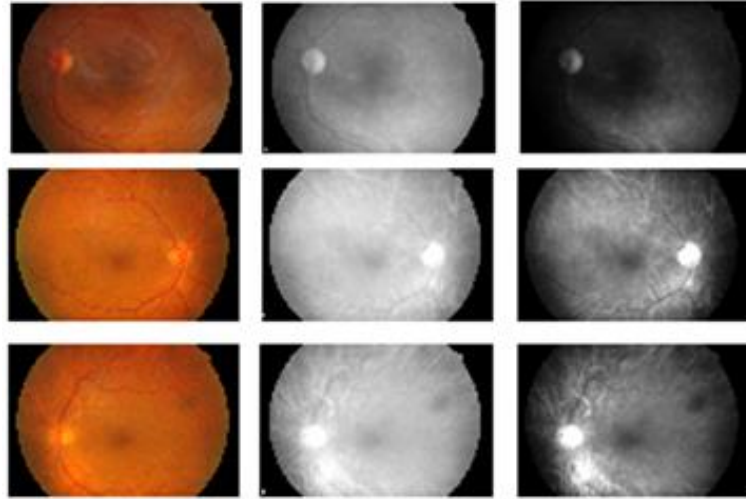

Fig.4. The three retinal images used as templates

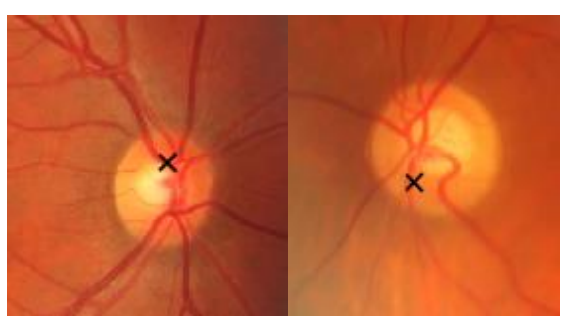

Fig.5 OD coordinates determined by template matching

\section{B. Active Contour Method}

The Active Contour Model (ACM) is a method used to segment the boundaries of an object on an image from other areas. It is the most commonly used model-based method for deformable models. In deformable models, segmentation is done by internal, external and image forces. In order to define the boundary of an OD, the closed curves must first be positioned close to the desired boundary. Then the number of curves is formed from the number of iterations. Internal forces ensure that the curve remains smooth throughout the deformation, while external forces guarantee that the curve is kept at a local minimum. Image forces ensure the curve is oriented towards apparent points like edges in the retinal image. The ACM is a method for semi-automatic segmentation by minimizing the energy function through iteration [20]. The path to form the curve is determined by minimizing the energy of the starting point selected by the user as being close to the optic boundary [21]. In other words, a curve is drawn inside or outside the OD; iterations are performed so that the curve takes the shape of the OD and segmentation is achieved by moving the curve by considering energy minimization $[21,22]$.

$$
E(v)=E_{i m g}(\vec{v})+E_{\text {int }}(\vec{v})+E_{\text {ext }}(\vec{v})
$$

where $E_{\text {int }}$ indicates the internal force caused by snakes. It is expressed by the tension and flexibility deformation of the contour. $E_{\text {img }}$ represents the image force, while $E_{\text {ext }}$ is the external force [23].

At the point where all forces are in balance, the position of ACM corresponds to the overall local minimum. In other words, the equation determines four boundary regions and minimizes the energy through orientation by external and image forces. The image energy equation in the $\mathrm{ACM}$ algorithm is given as follows:

$$
E_{\text {img }}(\vec{v})=-\int_{0}^{1} P(\vec{v}(s, t)) d s
$$

Where, $P(\vec{v}(s, t))$ shifts the ACM algorithm to the edges and is the magnitude of the image gradient [23].

$\nabla I(\vec{v}(s, t))$ prevents the algorithm moving towards lowerimage energy positions by smoothing the image and removing insignificant points. Furthermore, it orients the algorithm to more apparent details [23].

$$
\begin{aligned}
& \mathrm{P}(\overrightarrow{\mathrm{v}}(\mathrm{s}, \mathrm{t}))=|\nabla \mathrm{I}(\overrightarrow{\mathrm{v}}(\mathrm{s}, \mathrm{t}))| \\
& \mathrm{E}_{\text {int }}(\overrightarrow{\mathrm{v}})=\frac{1}{2} \int_{0}^{1} \alpha(\mathrm{s})\left|\frac{\partial \overrightarrow{\mathrm{v}}(\mathrm{s}, \mathrm{t})}{\partial \mathrm{s}}\right|^{2}+\beta(\mathrm{s})\left|\frac{\partial^{2} \overrightarrow{\mathrm{v}}(\mathrm{s}, \mathrm{t})}{\partial \mathrm{s}^{2}}\right|^{2} \mathrm{ds}
\end{aligned}
$$

where $\alpha(s)$ and $\beta(s)$ are the functions adjusting the stiffness and tension of the ACM algorithm. Contour strain is controlled by $\alpha(s)$, while tension is controlled by $\beta(s)$. Tension constraints are performed by the terms in the first line and make the ACM algorithm behave like a membrane. For stiffness, second-order terms are used to constrain the ACM algorithm to move like a thin layer. Thus, the balance of energies is achieved [23].

$$
E_{\text {ext }}(\vec{v})=\int_{0}^{1} P(\vec{v}(s)) d s
$$

The external force equation orients the algorithm image details for lines and edges in the retinal image [23].

$$
\begin{gathered}
E(\vec{v})=-\int_{0}^{1} P(\vec{v}(s, t)) d s+ \\
\frac{1}{2} \int_{0}^{1} \alpha(s)\left|\frac{\partial \vec{v}(s, t)}{\partial s}\right|^{2}+\beta(s)\left|\frac{\partial^{2} \vec{v}(s, t)}{\partial s^{2}}\right|^{2} d s
\end{gathered}
$$

This indicates the overall energy function of the ACM algorithm [23].

$$
\begin{aligned}
& \mathrm{E}(\overrightarrow{\mathrm{v}})=\int|\nabla \mathrm{I}(\mathrm{v})|^{2} \mathrm{ds}+\alpha(\mathrm{s}) \int\left|\left(\mathrm{v}_{\mathrm{s}}\right)\right|^{2} \mathrm{ds}+ \\
& \beta(\mathrm{s}) \int\left|\left(\mathrm{v}_{\mathrm{ss}}\right)\right|^{2} \mathrm{ds}
\end{aligned}
$$

where $\alpha(s)$ and $\beta(s)$ are weight parameters, $v_{s}$ is the first derivative with respect to curve parameter $s$, and the $v_{s s}$ parameter is the second derivative. In accordance with these equations, when the curve reaches the OD boundaries where high image gradients are present, the progression stops and the OD boundaries are determined [24-26].

\section{Performance Evaluation}

In the performance measurement of the proposed hybrid method, both OD localization and segmentation were evaluated separately. Whether localization was correct, was evaluated by template matching. Knowledge of a specialist physician was needed to evaluate the OD locations obtained by template matching. According to the specialist physician, all positions obtained by the template matching method were within the OD area, and the success rate was $100 \%$. In other 
words, the OD locations obtained from forty retinal images were correctly determined.

For segmentation of the OD, the positional information obtained by the template matching method was given as an input to the ACM. Then, the boundaries of the OD were determined by running the ACM. Expert physician skills were consulted to show that the segmented OD area was correctly determined. For each image, the OD areas were evaluated by the physician using the OD location and dimensions determined by the Active Contour. The Dice coefficient, accuracy, specificity and sensitivity were parameters used for performance evaluation. As a result of the process mentioned above, the OD obtained from a retinal image is shown in Figure 6.
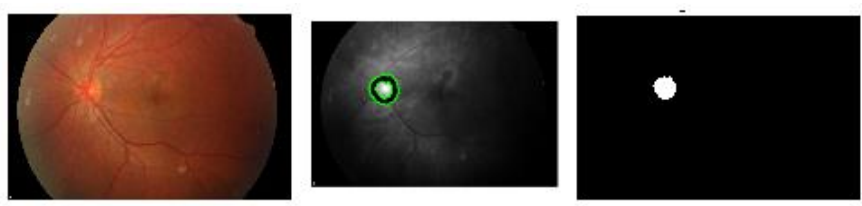

Fig.6. OD results segmented by using the ACM

The representative demonstration in Figure 7 was used to evaluate the OD area obtained manually by the physician from retinal images against the OD areas obtained by the ACM. The area $\mathrm{A}$ indicates the area segmented by the ACM, while the area $\mathrm{M}$ shows the actual OD area obtained by the physician. The intersecting area shows the OD area correctly determined by the applied method. The area of $A \cap M$ is indicated by True Positive (TP), $A-M$ False Positive and $M-A$ False Negative. The OD area obtained by the ACM was first evaluated using the Dice coefficient. Furthermore, the accuracy, specificity and sensitivity parameters defined by the confusion matrix can be evaluated whether or not the OD area was correctly determined.

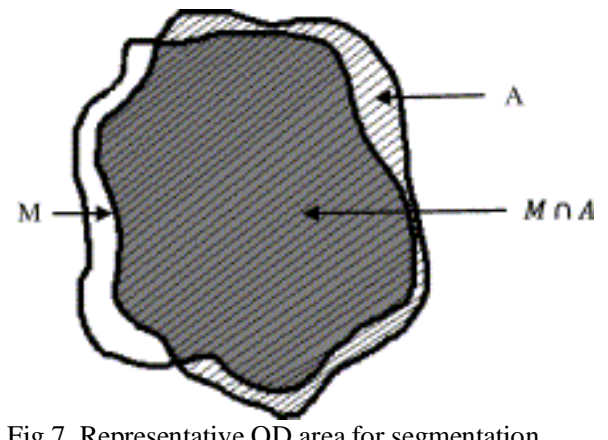

Fig.7. Representative OD area for segmentation

In addition to the Dice coefficient, the confusion matrix shown in Table 1 can be used to evaluate the performance of segmentation areas. In the confusion matrix, the rows show the OD areas determined by the physician and the columns indicate the OD areas determined by the Active Contour. The parameters $T P, T N, F P, F N$ given in the matrix are their values in pixels.

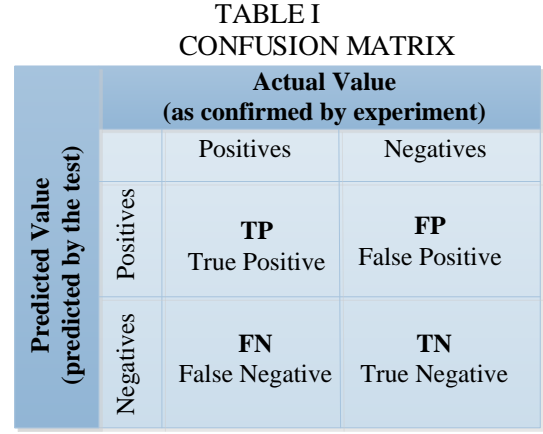

The most popular and simple parameters used to measure the performance of models in published literature are accuracy, specificity and sensitivity. Equations 11, 12 and 13 are used for the accuracy of the correctly determined OD area in terms of pixels.

$$
\text { Accuracy }=\frac{T P+T N}{T P+T N+F P+F N}
$$

The specificity given in Equation 12 is the ratio of the correctly determined pixel number to the OD area determined by the physician.

$$
\text { Specificity }=\frac{T P}{T P+T N}
$$

It is the ratio of the number of correctly classified positive samples to the overall number of positive samples.

$$
\text { Sensitivity }=\frac{T P}{T P+F N}
$$

Figure 8 shows accuracy, sensitivity and specificity changes obtained for forty retinal images.

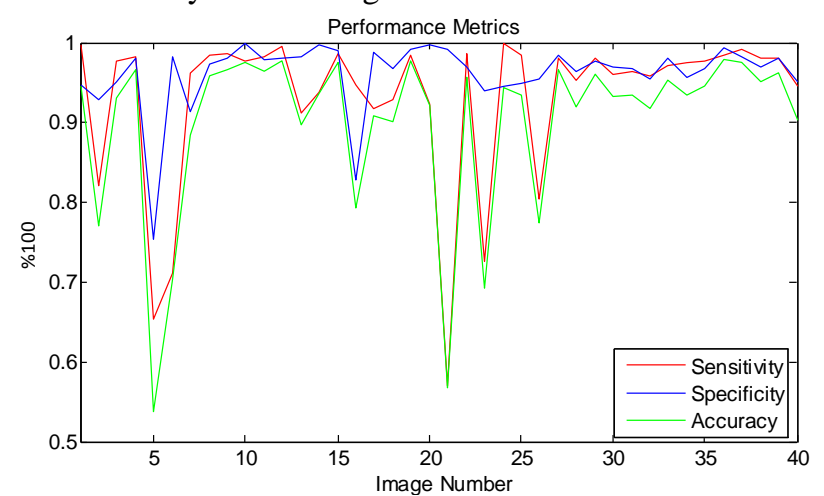

Fig.8. Accuracy, sensitivity and specificity values

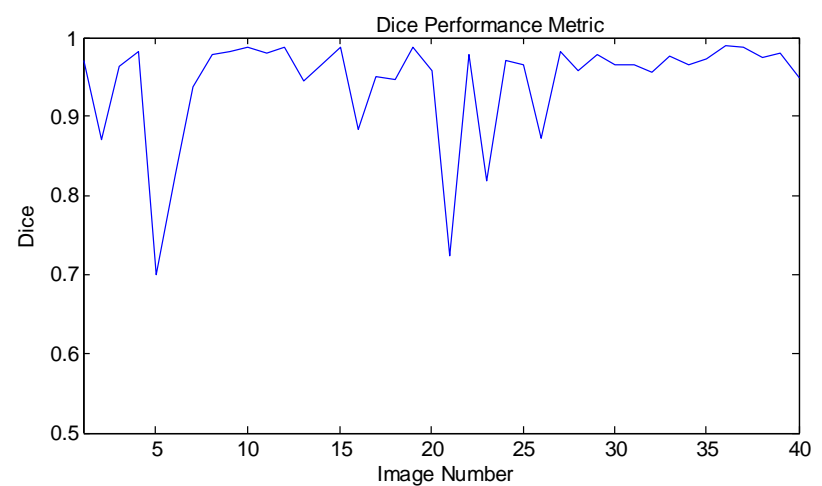

Fig.9. Dice coefficient for each retinal image 
The criteria of accuracy, specificity and sensitivity are not enough to obtain a meaningful comparison result. Therefore, it is also necessary to determine the Dice coefficient, known as the f-criterion. The Dice Similarity Coefficient takes a value between 0 and 1 . A value close to 1 means that the result is closest to that of the physician. According to published literature, a Dice coefficient greater than 0.7 indicates that the segmentation process is acceptable. Equation 14 shows the mathematical expression of the Dice coefficient, while Figure 9 shows the Dice coefficient change for fourth retinal images.

\section{PROPOSED ANDROID SYSTEM FRAMEWORK}

The Android-based system developed for the physician to monitor each patient using a mobile phone is shown in Figure 10. First, the OD is segmented by template matching and the Active Contour for each retinal image on the server. The OD sizes obtained manually and automatically are recorded in a database with the patient ID number. The physician can monitor the results of the OD by remotely connecting to the database with a mobile phone. The pseudo code for the application developed for this purpose is shown in Algorithm 1. The physician can link to the database on the internet (using a URL) with a username and password. If the connection is valid, the physician can access the results using the patient's ID number and add comments about the patient.

Figure 11 shows the developed Android-based interface and the results obtained from the database.

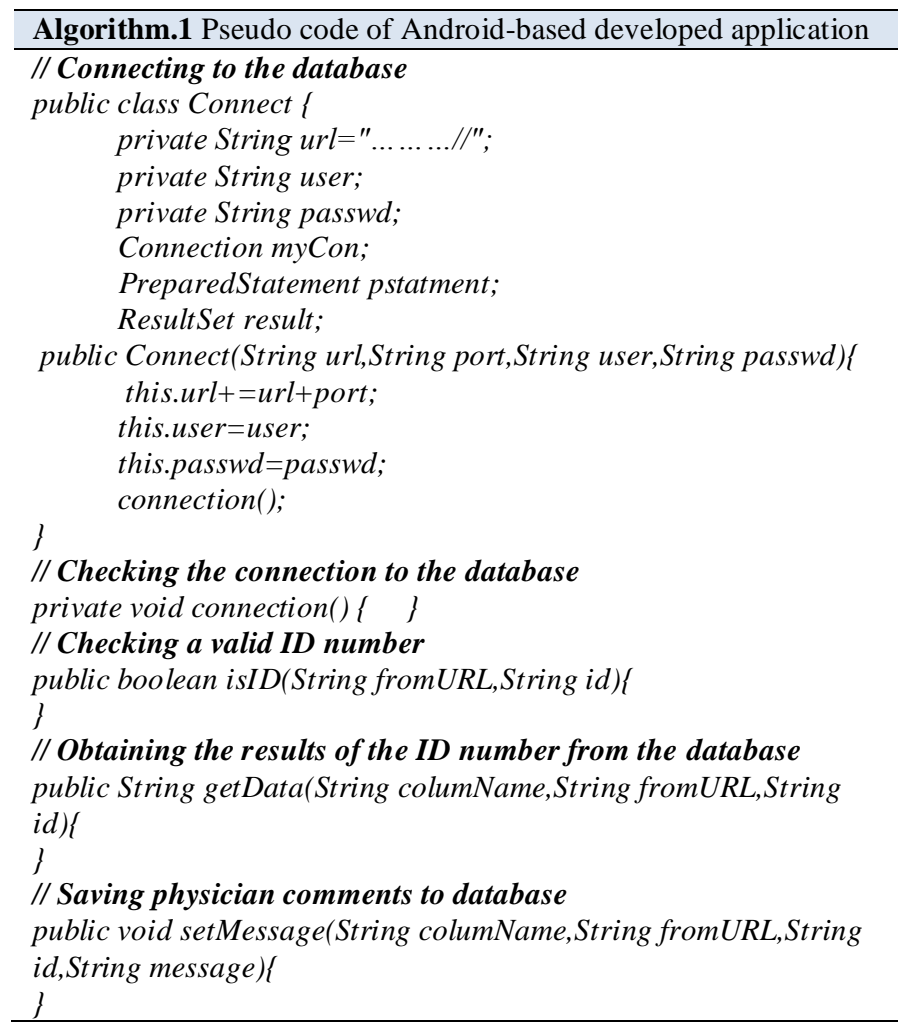

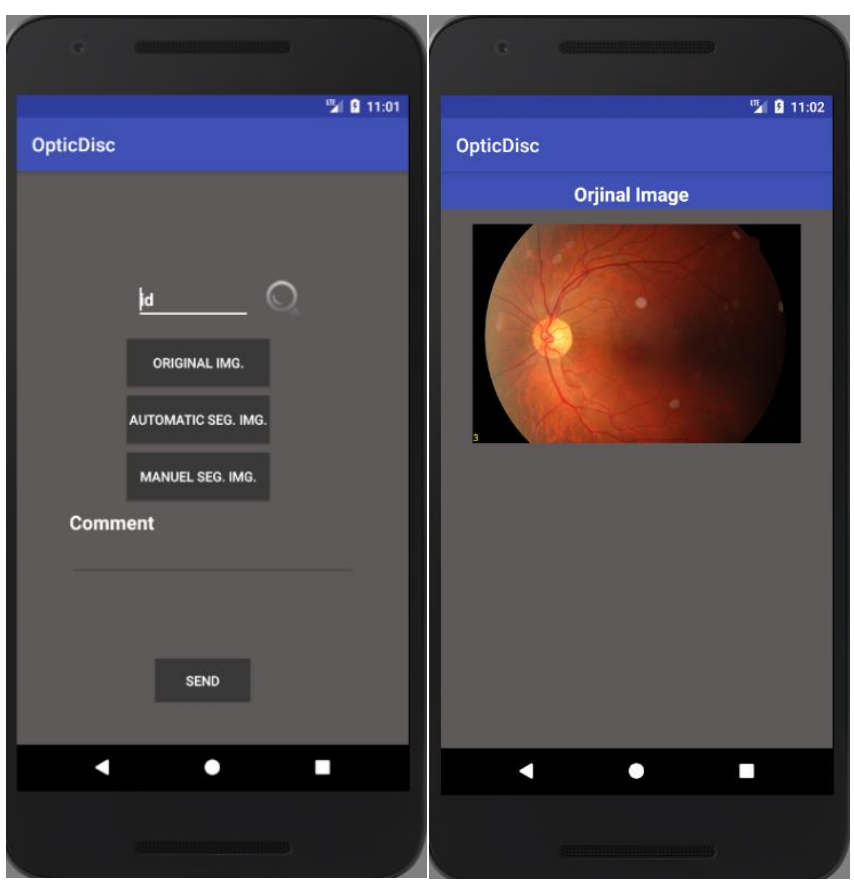

Fig.10. Android interface and a sample retinal image

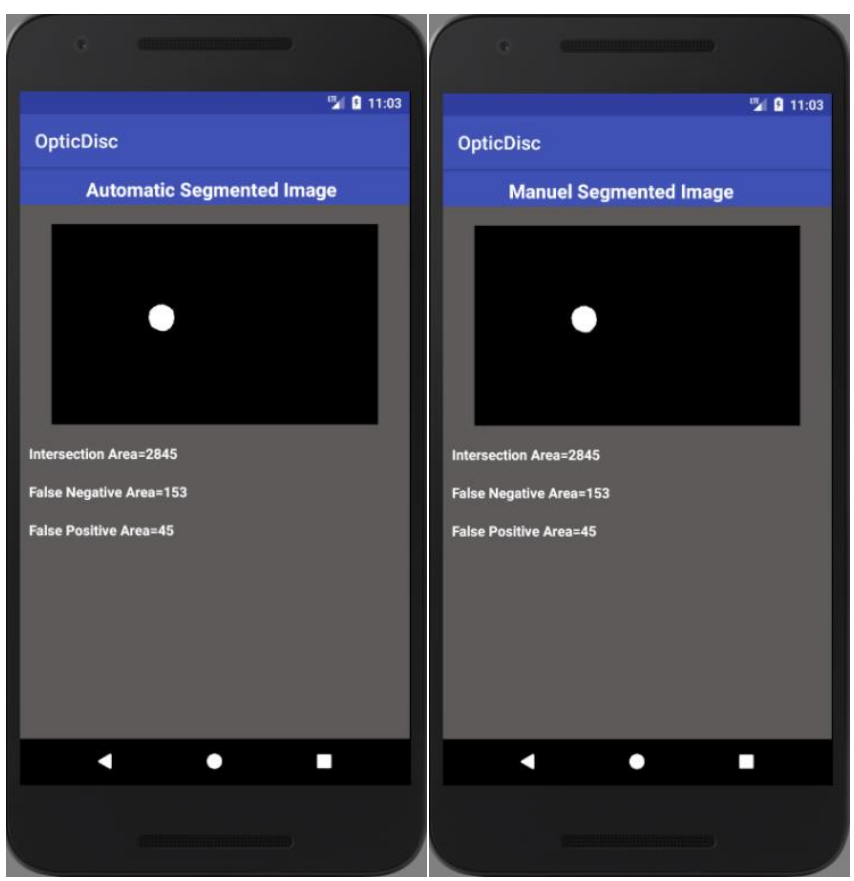

Fig.11. The results obtained from the database with the android system.

\section{DISCUSSION}

OD segmentation in retinal images is the first step in the diagnosis of diseases such as diabetic retinopathy, age-related macular degeneration, and glaucoma. The correct and precise segmentation of the OD will increase the correct diagnosis. In this paper, a hybrid method based on active contour and template matching is proposed for fully automatic segmentation of OD. According to the results obtained, the Dice coefficient 
was determined to be at the limit of its values in the 4th and 15th retinal images. The average Dice coefficient obtained for all images was 0.9432. Furthermore, the accuracy, specificity and sensitivity parameters were also determined to be less than 0.7 for the 4 th and 15 th images. The average values of these parameters for all images were calculated to be $0.90,0.961$ and 0.931 , respectively. As a result, because the average values of the parameters obtained by the Active Contour were calculated to be larger than 0.7 , the segmentation process was deemed to be acceptable. The OD segmentation results and literature comparisons are shown in Table 2.

TABLE.II

OD SEGMENTATION RESULTS and LITERATURE COMPARISONS

\begin{tabular}{|c|c|c|}
\hline Ref.No. & Methods & Success Rates \\
\hline [7] & $\begin{array}{c}\text { Component Analysis Method, } \\
\text { Stochastic Watershed } \\
\text { Algorithm }\end{array}$ & $\mathrm{A}=86.89 \%$ \\
\hline [8] & $\begin{array}{c}\text { Haar Wavelet } \\
\text { Transformation, } \\
\text { Hausdorff Based Matching }\end{array}$ & $A=93 \%$ \\
\hline [9] & $\begin{array}{l}\text { Sobel Edge Removal Filter, } \\
\text { Hough Transformation }\end{array}$ & $\mathrm{A}=97 \%$ \\
\hline$[10]$ & $\begin{array}{l}\text { L1 Minimization, } \\
\text { Region Growing }\end{array}$ & $\begin{array}{l}\mathrm{D}=92 \% \\
\mathrm{~J}=87 \%\end{array}$ \\
\hline [11] & Hybrid Vessel Phase Portrait & $\begin{array}{l}\mathrm{TN}=94.67 \% \\
\mathrm{TP}=98.13 \%\end{array}$ \\
\hline$[12]$ & $\begin{array}{l}\text { Active Contour, } \\
\text { Ellipse Fit Method }\end{array}$ & $A=98.67 \%$ \\
\hline$[13]$ & Histogram Matching & $\begin{array}{l}A=100 \% \\
A=98.9 \% \\
A=91.36 \%\end{array}$ \\
\hline [14] & Circular Transformation & $\begin{array}{c}A=98.77 \% \\
A=97.5 \% \\
A=99.75 \%\end{array}$ \\
\hline$[15]$ & $\begin{array}{l}\text { Brighness Based Method, } \\
\text { Supervised Descent Method }\end{array}$ & $\mathrm{O}=89.44 \%$ \\
\hline [16] & $\begin{array}{c}\text { Sparse Coding Technique, } \\
\text { Variotional model with } \\
\text { multiple Energies }\end{array}$ & $\begin{array}{l}\mathrm{O}=91 \% \\
\mathrm{O}=90 \% \\
\mathrm{O}=92 \%\end{array}$ \\
\hline$[17]$ & $\begin{array}{l}\text { Multiresolution Sliding Band } \\
\text { Filter }\end{array}$ & $\begin{array}{c}\mathrm{O}=89 \% \\
\mathrm{~A}=99.5 \%\end{array}$ \\
\hline$[18]$ & $\begin{array}{c}\text { Convolutional Neural } \\
\text { Network }\end{array}$ & $A=92.68 \%$ \\
\hline [19] & Sliding Window Technique & $\begin{array}{c}\mathrm{A}_{\min }=75 \% \\
\mathrm{~A}_{\max }=100 \%\end{array}$ \\
\hline $\begin{array}{l}\text { The } \\
\text { Proposed } \\
\text { Method }\end{array}$ & $\begin{array}{l}\text { Template Matching, } \\
\text { Active Contour }\end{array}$ & $\begin{array}{c}\mathrm{A}=900 \% \\
\mathrm{~S}=96.1 \% \\
\mathrm{Se}=\mathbf{9 3 . 1 \%} \\
\mathrm{D}=94.32 \%\end{array}$ \\
\hline
\end{tabular}

O:Overlapping, A: Accuracy, S:Specificity, Se:Sensitivity, D:Dice, $\mathrm{J}: J a c c a r d$

Different applied methods to OD segmentation were presented in Table.2. Some methods used a small dataset, while some used different datasets to train and test the algorithm. Methods were tested on both normal retinal images and on pathological images. The images in the data sets have different properties, so it may not be right to compare these methods with each other. Roughly, if we compare the proposed method to the other methods, the performance metrics are acceptable average level.

\section{CONCLUSION}

In preliminary steps for the detection of many diseases, such as glaucoma and diabetic retinopathy, locating the OD is important in diagnosing the disease. In this paper, OD segmentation was performed from the retinal images obtained from Firat University Ophthalmology Department. For this, a hybrid method was used to separate the OD from other areas of the eye. In this method, the OD location was determined by using the template matching algorithm. The obtained location information was used as input to the ACM, which is a semi-automated method. Thus, the ACM was converted to a fully automated method and the OD was segmented. Furthermore, An Android-based interface was prepared so that the physician could independently access the proposed hybrid method results from anywhere and add some comments. In the future, we intend to include the disease detection module to the android software interface. As a result, we think that the proposed software system can be used as a building block in developing an automated system for early detection of diseases.

\section{ACKNOWLEDGMENT}

The authors would like to thank Ophthalmologist Dr. Murat Firat for his help with the evaluation of the results.

\section{REFERENCES}

[1] Z. Yavuz, C. Ikibas, U. Şevik, C. Köse, "Retinal Görüntülerde Optik Diskin Otomatik Olarak Çıkartılması İçin Bir Yöntem", 5th International Advanced Technologies Symposium, Karabük, Türkiye, 13-15 Mayıs 2009, pp.8.

[2] J. Kaur, H.P. Sinha, "Automated localisation of optic disc and macula from fundus images", International Journal of Advanced Research in Computer Science and Software Engineering, 2(4), 2012, pp.242-249.

[3] C. İkibaş, 1Retinal İmgelerde Optik Disk ve Makulanın Tespiti ve Değerlendirilmesi”, PhD thesis, Karadeniz Teknik Üniversitesi, 2012.

[4] H. Li, O. Chutatape, "Automatic location of optic disc in retinal images", International Conference on Image Processing, Thessaloniki, Greece, 2001, pp. 837- 840 .

[5] A. Osareh, M. Mirmehdi, B. Thomas, R. Markham, "Colour Morphology and Snakes for Optic Disc Localisation", 16th IEEE Int. Conf. Pattern Recognition, vol.1, 2002, pp. 743-746.

[6] A. Ahmed, B. Ritambhar, R. Kaamran, L. Vasudevan, "'Optic Disc and Optic Cup Segmentation Methodologies for Glaucoma Image Detection: A Survey", Journal of Ophthalmology, Article ID 180972, 2015, pp.28.

[7] S. Morales, V. Naranjo J. Angulo M. Alcañiz, "Automatic Detection of Optic Disc Based on PCA and Mathematical Morphology", IEEE Transactions on Medical Imaging, 32(4), 2013, pp. 786-796.

[8] M. Lalonde, M. Beaulieu, L. Gagnon, "Fast and robust optic disc detection using pyramidal decomposition and hausdorff-based template matching”, Medical Imaging, IEEE Transactions, 20(11), 2001, pp. 1193 -1200 . 
[9] T. Chaichana, S. Yoowattana, et al, "Edge detection of the optic disc in retinal images based on identification of a round shape", International Symposium Communications and Information Technologies, 2008, pp. $670-674$.

[10] S.A. Tuncer, T. Selçuk M. Parlak, A. Alkan, "'Hybrid approach optic disc segmentation for retinal images", International Artificial Intelligence and Data Processing Symposium (IDAP), 2017, pp.1-6.

[11] N. Muangnak, P. Aimmanee, S. Makhanov, "Automatic optic disc detection in retinal images using hybrid vessel phase portrait analysis",Med Biol Eng Comput., 56(4), 2018, pp.583-598.

[12] R. Fulonga L. Wei, Y. Jinzhua et.al, "Automatic optic disc localization and segmentation in retinal images by a line operator and level sets", Technology and Health Care, 24(2), 2016, pp. S767-S776.

[13] A. Dehghani, H. A. Moghaddam, M. S. Moin, "Optic disc localization in retinal images using histogram matching", EURASIP Journal on Image and Video Processing, 2012, 2012(19).

[14] S. Lu, "Accurate and efficient optic disc detection and segmentation by a circular transformation", IEEE Transactions on Medical Imaging, 30(12), 2011, pp. 2126-2133.

[15] A. Li, Z. Niu, J. Cheng, et.al., "Learning supervised descent directions for optic disc segmentation", Neurocomputing, 275, 2018, pp.350-357.

[16] B. Dai, X. Wu, W. Bu, "Optic disc segmentation based on variational model with multiple energies", Pattern Recognition, 64, 2017, pp. 226235.

[17] B. Dashtbozorg, A. Mendonça, M.A. Campilho, "Optic disc segmentation using the sliding band filter", Computers in Biology and Medicine, 56, 2015,pp. 1-12.

[18] J. H. Tan, U.R. Acharya, S.V. Bhandary et. al. "Segmentation of optic disc, fovea and retinal vasculature using a single convolutional neural network", Journal of Computational Science, 20, 2017, pp.70-79.

[19] L.A. Muhammed, "Localizing Optic Disc in Retinal Image Automatically with Entropy Based Algorithm", International Journal of Biomedical Imaging, Article ID 2815163, 2018, pp.7.

[20] T.F. Chan, L.A. Vese, "Active contours without edges", IEEE Transactions on Image Processing, 10(2), 2001.

[21] M. Kass, A.Witkin, D. Terzopoulos, "Snakes: active contour models", International Journal of Computer Vision, 1, 1988, pp.321-331.

[22] P.P.R. Filho, P.C. Cortez, A.C. da S. Barros, et.al., "Novel Adaptive Balloon Active Contour Method based on internal force for image segmentation A systematic evaluation on synthetic and real images", Expert Systems with Applications, 41, 2014, pp.7707-7721.

[23] T.F. Chan, L.A. Vese, "Active contours without edges", IEEE Transactions on Image Processing, 10(2), 2001.

[24] E. Isıkc1, D.G. Duru, "Multiple Skleroz Manyetik Rezonans Görüntülerinde Aktif Kontur Modeli ile Lezyon Tespiti”, Tip Teknolojileri Ulusal Kongresi, Muğla,Türkiye, 2015.

[25] S.A. Tuncer, A. Alkan, "Segmentation of thyroid nodules with K-means algorithm on mobile devices", 16th IEEE International Symposium on Computational Intelligence and Informatics, Budapest, Hungary, 2015, pp. 345-348.

[26] A. Alkan, S.A. Tuncer, M. Gunay, "Comparative MR image analysis for thyroid nodule detection and quantification", Measurement, 47, 2014, pp. 861-868.

[27] M. Niemeijer, B. van Ginneken, F. Ter Haar et.al., "Automatic detection of the optic disc, fovea and vascular arch in digital color photographs of the retina", Proceedings of the British Machine Vision Conference 2005, 109-118.

\section{BIOGRAPHIES}

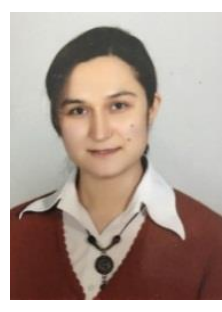

Seda ARSLAN TUNCER was born in Kahramanmaraş, Turkey 1983. She received the B.S. and M.S. degree in computer engineering from the Firat University in Elazig 2005 and 2008 respectively. She received the Ph.D. degrees in electricalelectronics engineering from Kahramanmaras Sutcu Imam University in 2014. From 2010 to 2014, she was a lecturer at department of informatics, Firat University, Elazig, Turkey. Since 2014, she has been an Assistant Professor at software engineering. Her research interest include biomedical, image and signal processing, embedded systems, random numbers generators. 